\title{
ASS für die kardiovaskuläre Prävention?
}

\author{
Die primäre Prävention kardiovaskulärer Erkrankungen ist bei Diabetikern von großer \\ Bedeutung, da Inzidenz und Sterblichkeit bei ihnen erhöht sind. Eine Studie legt nun \\ nahe, dass dabei die Zugabe von Acetylsalicylsäure nützlich sein könnte.
}

_ Seit 2015 wird die Zugabe von 75-162 $\mathrm{mg} / \mathrm{d}$ Acetylsalicylsäure (ASS) in den Leitlinien der American Diabetes Association als primäre Präventionsstrategie empfohlen. Dies betrifft Patienten mit erhöhtem kardiovaskulärem Risiko einschließlich Männer > 50 Jahren und Frauen $>60$ Jahren mit wenigstens einem bedeutenden kardiovaskulären Risikofaktor. Die Evidenzbasis war bisher allerdings schwach. Nun untersuchte eine Studie den Nutzen der Zugabe von $100 \mathrm{mg} / \mathrm{d}$ ASS anhand der Serumspiegel neuer Biomarker für erhöhtes kardiovaskuläres Risiko - etwa CD40Ligand (sCD40L) und Serum-Myeloperoxidase (MPO).
Einbezogen wurden 213 ambulante Patienten im mittleren Alter von 57,7 Jahren mit Übergewicht (mittlerer BMI: 27,7), Hypertonie (140-180/90-105 $\mathrm{mmHg}$ ), LDL-Cholesterin-Werten $<160$ $\mathrm{mg} / \mathrm{dl}, \mathrm{HbA}_{\mathrm{lc}}$-Werten $<7,5 \%$ in primärer Prävention. Keiner hatte bisher Blutdrucksenker oder ThrombozytenAggregationshemmer erhalten. Alle hielten eine kontrollierte Diät ein und nahmen an Bewegungsprogrammen teil.

Die Patienten wurden doppelblind randomisiert und erhielten täglich $5 \mathrm{mg}$ Amlodipin entweder allein oder in Kombination mit $100 \mathrm{mg}$ ASS. Im Mittel lagen die Blutdruckwerte zu Beginn bei 156/96 mmHg bzw. 154/97 mmHg.

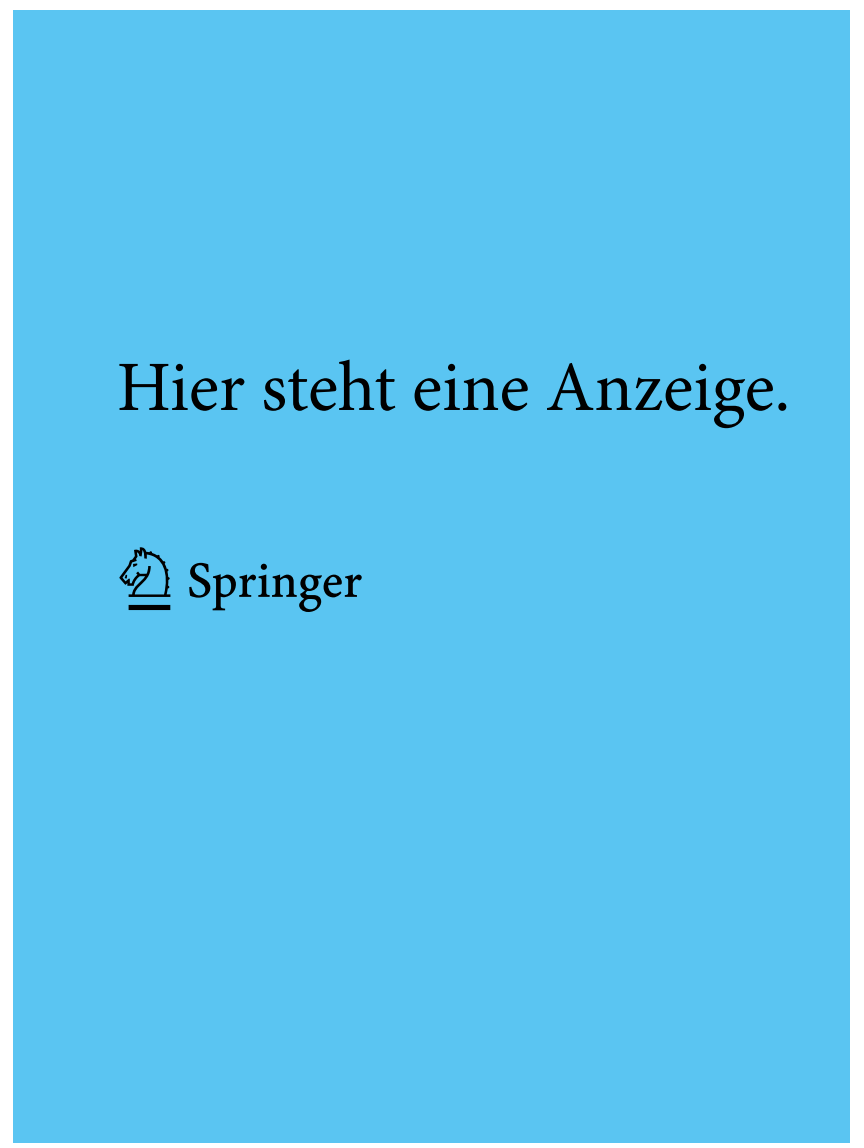

Nach der dreimonatigen Phase A hatten 110 Patienten noch Blutdruckwerte $>$ 140/90 mmHg, sodass bei ihnen die Amlodipin-Dosis für weitere drei Monate (Phase B) verdoppelt wurde.

Der systolische Blutdruck sank ohne Unterschied zwischen den Gruppen unter $5 \mathrm{mg}$ Amlodipin um ca. 6\% und unter $10 \mathrm{mg}$ um 13\%. Nur Patienten mit ASS-Zugabe verzeichneten nach Phase A einen signifikanten Abfall von MPO $(-9,7 \%)$ und sCDL40 (-15,7\%) im Vergleich zum Ausgangswert und im Vergleich zu Amlodipin ohne ASS (-5,1 bzw. $-13,6 \%)$. Auch nach Phase B waren nur bei ASS-Zugabe MPO (-9,2\%) und sCDL40 (-20\%) signifikant abgefallen.

Parallel kam es in beiden Phasen nur unter ASS-Zugabe zu einem signifikanten Abfall von hoch sensitivem CRP (-15 bzw. $-19 \%)$ und TNF-alpha (-21,7 bzw. $-15 \%)$ sowie zu einem signifikanten Anstieg von Adiponektin (15 bzw. 12\%).

- Derosa G et al. A study about the relevance of adding ace tylsalicylic acid in primary prevention in subjects with type 2 diabetes mellitus: effects on some new emerging biomarkers of cardiovascular risk. Cardiovasc Diabetol. 2015;14:95

\section{KOMMENTAR}

Die Ergebnisse legen nahe, dass die Zugabe von ASS zur Amlodipin-Therapie in der Primärprävention bei Diabetikern mit Hypertonie nützlich sein könnte, da inflammatorische Marker und neuere Biomarker, die zur kardiovaskulären Risikostratifizierung als geeignet erscheinen, stärker reduziert wurden. Patienten mit Hypertonie haben einer früheren Studie zufolge erhöhte Konzentrationen von SCD40L und MPO [Derosa G et al. Curr Med Res Opin. 2012;28:1435-45]. Blutdrucksenkung per se hatte auf diese Marker einen günstigen Effekt.

Die Studie stärkt allgemein die Rolle von ASS bei der Reduktion von Inflammation und Endothelschäden, auch wenn noch offen ist, ob die Senkung der Biomarker tatsächlich das kardiovaskuläre Risiko reduziert.

Prof. Dr. med. E. Fritschka 